\title{
CONTRIBUCIÓN DE LA ERGONOMÍA A LA SEGURIDAD DEL PACIENTE: APLICACIÓN DE LA LISTA DE VERIFICACIÓN ERGONÓMICA PARA EL SECTOR DE LA SALUD EN UN HOSPITAL UNIVERSITARIO DE COLOMBIA
}

\author{
CONTRIBUTION OF ERGONOMICS TO PATIENT SAFETY: APPLICATION OF THE \\ ERGONOMIC CHECKPOINTS IN HEALTH CARE WORK IN A UNIVERSITY HOSPITAL IN \\ COLOMBIA
}

\author{
Yaniel Torres* \\ Yordán Rodríguez ${ }^{* *}$ \\ Néstor Raúl Buitrago ${ }^{* * *}$
}

\begin{abstract}
Resumen: La integración de la ergonomía en el diseño y la optimización de los sistemas de salud es un elemento esencial para mejorar la seguridad del paciente. En este artículo se presentan los resultados de la aplicación, en un hospital de Colombia, de la lista de verificación ergonómica para el sector de la salud, propuesta por la Asociación Internacional de Ergonomía. Para la aplicación de esta lista de verificación se contó con la participación de 16 enfermeras y dos supervisores de la sala de hospitalización general. Del total de 60 puntos contenidos en la lista de verificación, se identificaron 22 puntos de mejora, los cuales estuvieron asociados al almacenamiento y manejo de materiales (4 puntos), al manejo seguro de pacientes (3 puntos), a los puestos de trabajo (3 puntos), al control de infecciones (3 puntos) y a la organización del trabajo (3 puntos). Este estudio muestra cómo la aplicación de la ergonomía, mediante el uso de listas de verificación prácticas y empleando un enfoque participativo, puede ayudar a mejorar la seguridad del paciente y la seguridad laboral en organizaciones del sector de la salud (hospitales, clínicas, consultorios, etc.). Los resultados respaldan la utilidad de aplicar principios ergonómicos de amplio alcance y de fácil comprensión. La aplicación de la lista de verificación propuesta por la Asociación Internacional de Ergonomía representa una novedad en el ecosistema de salud colombiano y abre la puerta a futuros desarrollos, apoyando así el establecimiento de estrategias que incluyan a la ergonomía como pilar fundamental para la mejora de la seguridad del paciente.
\end{abstract}

Palabras clave: Ergonomía, lista de verificación ergonómica para el sector de la salud, hospital universitario, seguridad del paciente, seguridad laboral, sistema de salud.

\footnotetext{
Abstract: The integration of ergonomics into the design and optimization of healthcare systems is an essential element for improving patient safety. This article presents the application results in a

${ }^{*}$ Department of Mechanical Engineering, École de Technologie Supérieure. Montreal, Canadá. Correo electrónico: yaniel.torres-medina.1@ens.etsmtl.ca. Orcid: https://orcid.org/oooo-0002-9825-9437. Autor de correspondencia.

${ }^{* *}$ Facultad Nacional de Salud Pública, Universidad de Antioquia. Medellín, Colombia. Correo electrónico: yordan.rodriguez@udea.edu.co. Orcid: https://orcid.org/oooo-0002-0079-4336

${ }^{* * *}$ Facultad Nacional de Salud Pública, Universidad de Antioquia. Medellín, Colombia. Correo electrónico: nestor.buitrago@udea.edu.co. Orcid: https://orcid.org/oooo-00o2-2229-1684
} 
Colombian hospital of the Ergonomic Checkpoints in Health Care Work proposed by the International Ergonomics Association (IEA). The application of the guidelines was carried out with the participation of 16 nurses and two supervisors. Out of the 60 checkpoints contained in the IEA document, 22 points for improvement were identified. The identified checkpoints were associated with the storage and handling of material (4 points), safe handling of patients (3 points), workstations ( 3 points), infection control (3 points) and work organization (3 points). This study shows how applying ergonomics by using practical guidelines/tools, and a participatory approach can help to potentially improve patient safety in healthcare organizations (hospitals, clinics, medical centers, etc.). The results support the usefulness of applying ergonomic principles that are broad in scope and easy to understand. The application of the guidelines proposed by the IEA represents a novelty in the Colombian healthcare ecosystem. It opens the door to future developments, thus supporting the establishment of strategies that include ergonomics as a fundamental pillar for improving patient safety.

Keywords: Ergonomics, ergonomic checkpoints in health care work, university hospital, patient safety, occupational safety, healthcare system.

Recepción: 17.05.2021 / Revisión: 21.05.2021 / Aceptación: 04.08.2021

\section{Introducción}

La ergonomía, también conocida como factores humanos, es una de las disciplinas científicas de mayor contribución al diseño de sistemas de trabajos seguros y eficientes, incluyendo los sistemas de salud (Hignett et al., 2013; Holden et al., 2013; Rodríguez \& Hignett, 2021). El objetivo de la ergonomía es diseñar sistemas de trabajo que optimicen el bienestar humano y el rendimiento global del sistema (seguridad, bienestar, calidad y productividad, entre otros) (International Ergonomics Association [Asociación Internacional de Ergonomía, IEA], 2000). Para ello, el enfoque de la ergonomía pone al humano en el centro, tomando en cuenta sus capacidades y limitaciones tanto físicas como cognitivas, a la hora de diseñar el sistema de trabajo. La aplicación del enfoque propuesto por la ergonomía a los sistemas de salud puede impactar notablemente la seguridad del personal de la salud, los pacientes y sus familiares. Por ejemplo, en un estudio realizado a finales de 2019 por el National Health Service (Servicio Nacional de Salud, NHS) del Reino Unido, se seleccionó a la ergonomía como la temática de mayor prioridad en la estrategia de seguridad del paciente de los próximos años en ese país (NHS, 2018).

La Organización Mundial de la Salud (OMS) define la seguridad del paciente como "la ausencia de daños prevenibles para el paciente durante el proceso de atención de salud y la reducción del riesgo de daños innecesarios [...] a un mínimo aceptable"(World Health Organization, WHO, 2019a). Para este organismo internacional, la seguridad del paciente representa un tema prioritario en el marco de sus planes estratégicos, con el objetivo de mejorar los sistemas de salud a escala global (WHO, 2017). El propósito principal de la seguridad del paciente es prevenir los eventos adversos que podrían resultar en daños a los pacientes durante el proceso de atención a la salud. Los eventos adversos incluyen los errores médicos, pero también las infecciones intrahospitalarias y las caídas de los pacientes.

Las estadísticas a escala global muestran que los errores médicos ocasionan alrededor 
de dos tercios del total de los daños a los pacientes (WHO, 2017). Por ejemplo, en Estados Unidos de América, los errores médicos causan la muerte a un poco más de 250000 pacientes cada año y constituyen la tercera causa de muerte (Makary \& Daniel, 2016). En el caso de Canadá, se estima en alrededor de 30 ooo el número de pacientes que pierden la vida cada año por errores médicos, siendo igualmente la tercera causa de muerte (Pisters, 2015). En la Unión Europea, los errores médicos y los eventos adversos en general se producen en hasta el 12 \% del total de las hospitalizaciones (WHO, 2019b), mientras que, en el Reino Unido, representan alrededor del 10 \% de las hospitalizaciones (Donaldson, 2002).

En países en vías de desarrollo, es difícil cuantificar y consolidar con precisión las estadísticas sobre los errores médicos u otros eventos adversos. Con frecuencia, las estadísticas son solamente reportadas en estudios aislados llevados a cabo por compañías aseguradoras, hospitales o farmacias (Aranaz-Andrés et al., 2011; Machado-Alba et al., 2016; Moscoso et al., 2015). Sin embargo, la OMS, en uno de sus informes, hace referencia a un estudio que se llevó a cabo en 26 países de bajos y medios ingresos, en el cual la tasa de eventos adversos fue de alrededor del $8 \%$, de los que el $83 \%$ pudieron haberse prevenido y el 30 \% llevaron a la muerte (WHO, 2017). Según este mismo reporte de la OMS, una de cada diez hospitalizaciones termina ocasionando daños al paciente.

La ergonomía ha sido adoptada como práctica para mejorar la seguridad del paciente en varios sistemas de salud de países industrialmente avanzados (Blouin et al., 2016; Carayon et al., 2014). Sin embargo, la aplicación de la ergonomía en los países en vías de desarrollo se ha centrado, con mayor énfasis, en los elementos físicos del trabajo: uso de herramientas, diseño de puestos de trabajo, manipulación manual de materiales y evaluación de los entornos de trabajo, especialmente en climas tropicales (Huck-Soo \& Richardson, 2012). Por otro lado, los sistemas de salud en países en vías de desarrollo enfrentan diversos desafíos, teniendo en cuenta la limitada disponibilidad de infraestructura y la falta general de recursos y profesionales sanitarios, en comparación con los países industrialmente avanzados (Organisation for Economic Co-operation and Development, 2018). Por las razones descritas anteriormente, Aceves-González et al. (2020) reconocen que la aplicación de la ergonomía en los sistemas de salud de países en vías de desarrollo ha sido limitada, como es el caso de América Latina.

Una alternativa a esta situación es el uso de estrategias participativas de bajo coste. Este enfoque se ha explorado anteriormente, siendo un ejemplo de ello la lista de verificación ergonómica, desarrollada y publicada en 1996 (primera edición) por la Organización Internacional del Trabajo (International Labour Organization) y la IEA, y enfocada en la industria general (2010). Posteriormente, en el año 2017, tomando como referencia la experiencia en el uso de esta lista de verificación, la Human Ergology Society y la IEA proponen una nueva específica para el sector sanitario (Ergonomic Checkpoints in Health Care), con el objetivo de fomentar la aplicación práctica de la ergonomía en un formato relativamente fácil de usar y participativo (IEA, 2017).

Por tanto, el objetivo de este artículo es presentar los resultados de la aplicación de esta última lista de verificación ergonómica en la sala de hospitalización general de un hospital universitario en Colombia. De esta manera, se busca identificar las condiciones del sistema de trabajo que podrían conducir a la ocurrencia de errores de medicación y que pueden ser 
mejoradas, y así ayudar a mejorar la seguridad del paciente.

\section{Materiales y métodos}

Se realizó un estudio descriptivo transversal en la sala de hospitalización general de un hospital universitario de Medellín, Colombia. Esta unidad de servicio cuenta con 116 camas activas.

Un grupo de enfermeras profesionales $(\mathrm{n}=16)$ participó en el estudio: ocho auxiliares y ocho licenciadas. El estudio se llevó a cabo bajo un enfoque de intervención participativa. Se hicieron entrevistas grupales por la mañana, durante la reunión de cambio de turno, cuando se reúnen las enfermeras y los supervisores. Se obtuvo el consentimiento informado y por escrito de los participantes.

El trabajo de campo fue realizado por dos estudiantes de la Especialización en Ergonomía que ofrece la Facultad Nacional de Salud Pública de la Universidad de Antioquia en Medellín, Colombia. Uno de los estudiantes actuó como facilitador, bajo la estrecha supervisión de profesionales experimentados en ergonomía.

Para la investigación, se utilizó la lista de verificación ergonómica para el sector sanitario propuesta por la IEA (2017) como documento marco para llevar a cabo las reuniones grupales. Así, se identificaron de manera estructurada las áreas de mejora en el sistema de trabajo. Igualmente, se revisaron los procedimientos y protocolos laborales, para obtener información global acerca de la ejecución de las tareas desde el punto de vista del trabajo prescrito. También se utilizó la observación de terreno como complemento, para ganar mejor comprensión del trabajo real efectuado por las enfermeras. Además, se tomaron fotografías, con el fin de identificar e ilustrar visualmente las oportunidades de mejora en el sistema de trabajo analizado (sala de hospitalización).

\section{Lista de verificación ergonómica para el sector de la salud}

La lista de verificación ergonómica para el sector de la salud propuesta por la IEA es un documento de dominio público que recopila diversas acciones de mejora enfocadas en la práctica (IEA, 2017). Aunque se presentan desde la perspectiva de la ergonomía, también incluyen distintos aspectos asociados a la seguridad y la salud en el trabajo. Esto se debe a que la ergonomía no está disociada de otras problemáticas en los contextos de trabajo objeto de estudio.

Esta lista incluye un total de 60 puntos de verificación, los cuales están agrupados en 10 categorías (seis puntos de verificación por cada categoría). Las diez categorías incluidas en el documento son: 1) almacenamiento y manipulación de materiales, 2) seguridad de las máquinas y herramientas manuales, 3) manipulación segura de los pacientes, 4) puestos de trabajo, 5) entorno físico, 6) substancias y agentes peligrosos, 7) control de infecciones, 8) instalaciones de descanso y bienestar, 9) plan de evacuación y emergencia, 10) organización del trabajo y seguridad de los pacientes. 
En todo el documento se hace hincapié en la presentación de mejoras sencillas y de bajo coste que sean factibles de aplicar tanto en los países industrializados como en los países en vías de desarrollo. Los puntos de verificación están diseñados para que sean fáciles de aplicar y adaptables a diversas situaciones de trabajo. Todos los puntos de verificación se acompañan de imágenes y un texto explicativo, lo que facilita su comprensión incluso para el personal no experto en temas de ergonomía.

La figura 1 muestra dos ejemplos de imágenes asociadas al punto de control 2 y al punto de control 4, respectivamente. Ambos puntos de verificación fueron identificados como puntos de mejora en el estudio.

Figura 1. Puntos de verificación objeto de mejora.
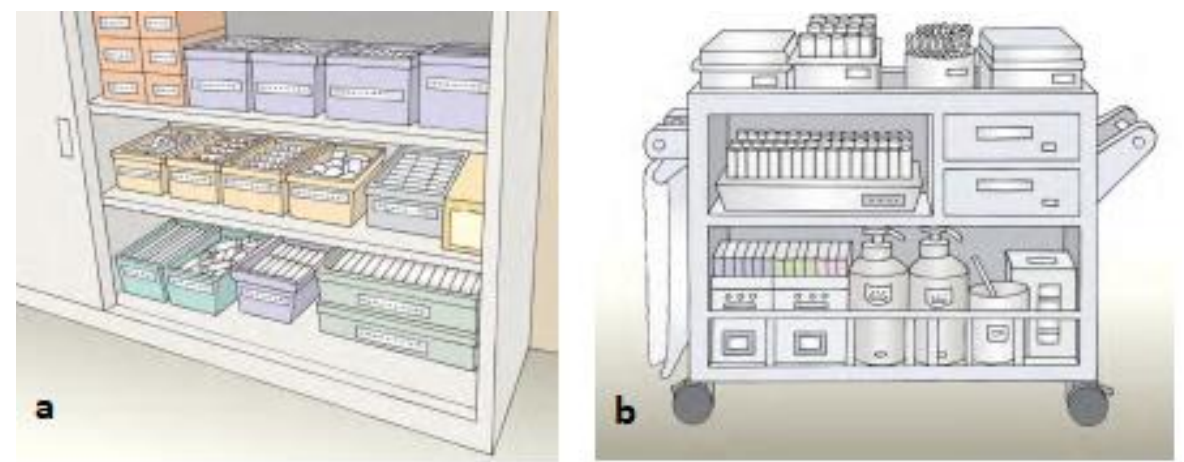

Nota. a. Punto de control 2: el uso de pequeños contenedores y etiquetas facilita el almacenamiento; b. punto de control 4: carrito bien organizado para el material de tratamiento médico. Fuente. IEA (2017).

\section{Resultados y discusión}

Del total de los 60 puntos propuestos por el documento de la IEA, en esta investigación se identificaron 22 puntos susceptibles de mejoras. Las categorías identificadas fueron: almacenamiento y la manipulación de materiales (4 puntos), la manipulación segura de los pacientes (3 puntos), los puestos de trabajo (3 puntos), el control de las infecciones (3 puntos) y la organización del trabajo y seguridad de los pacientes (3 puntos). Se identificaron cinco puntos prioritarios, tres en la categoría de almacenamiento y manipulación de materiales, y uno en la categoría de organización del trabajo y seguridad del paciente. Estos puntos prioritarios fueron: 1) utilizar estantes o estanterías de varios niveles y contenedores pequeños para minimizar el transporte manual de los materiales; 2) hacer que las etiquetas y las señales sean fáciles de ver, de leer y de entender; 3) utilizar carros, carretillas de mano y otros dispositivos con ruedas, convenientes para trasladar los materiales, y 4) promover servicios sanitarios más seguros y una cultura de seguridad del paciente que implique al personal, la dirección y los pacientes.

En la tabla 1 se muestran algunos puntos de mejora identificados con la ayuda de la lista de verificación ergonómica para el sector salud. 
Tabla 1. Ejemplos de puntos de verificación susceptible de mejoras identificados durante la investigación y agrupados por categoría.

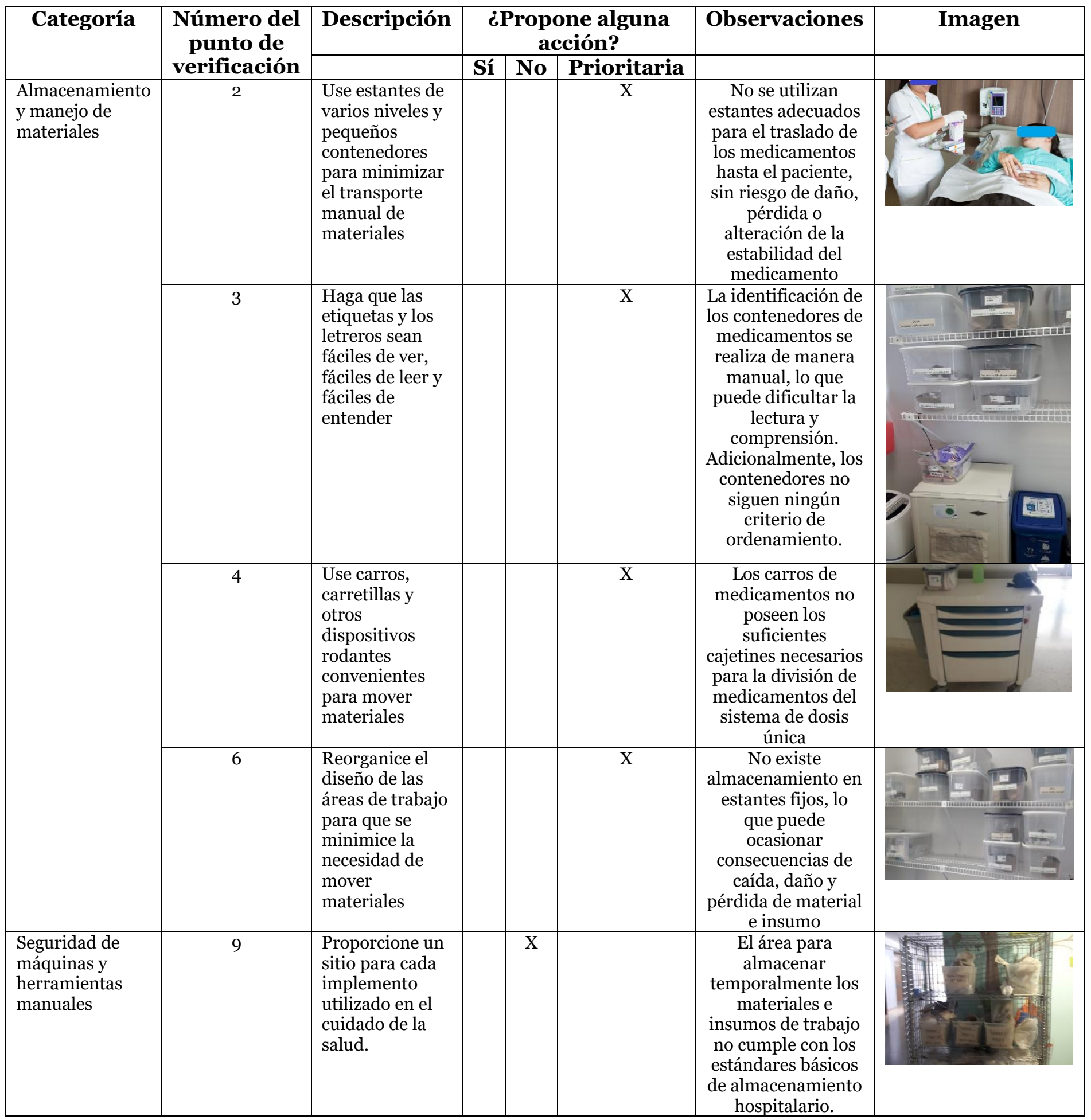




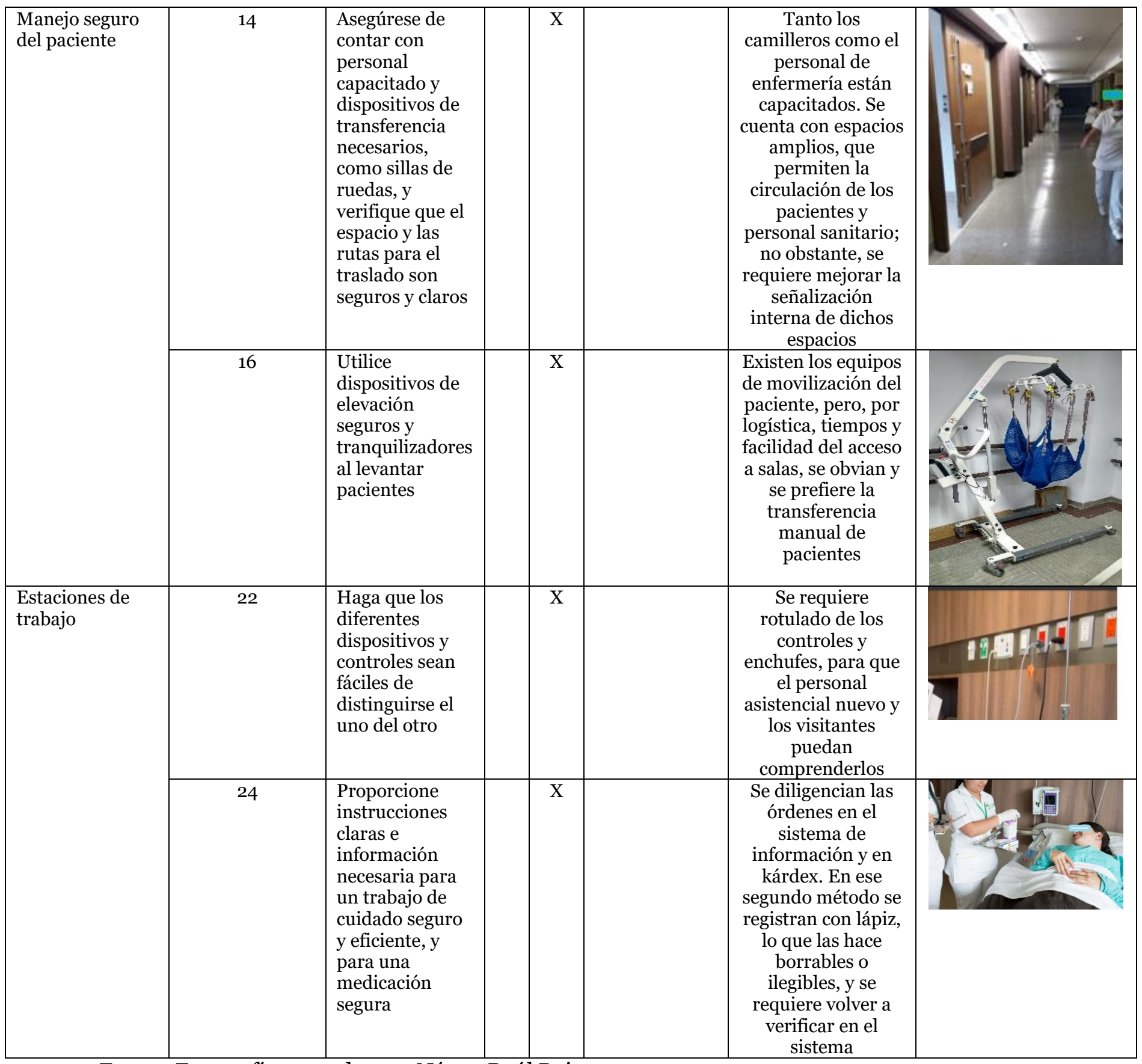

Fuente. Fotografías tomadas por Néstor Raúl Buitrago.

Esta investigación constituye una experiencia relativamente novedosa en el contexto hospitalario colombiano, en el que se movilizaron varios actores en torno al tema de la seguridad del paciente desde la perspectiva de la ergonomía. La lista de verificación utilizada es un documento relativamente nuevo (Kogi et al., 2019) y, hasta donde se sabe, aún no han sido reportadas, en la literatura, aplicaciones de esta en Colombia. No obstante, estrategias similares han demostrado ser efectivas en países en vías de desarrollo en contextos diferentes al de la salud, por ejemplo, en la agricultura, la industria en general y la pequeña empresa (Budnick et al., 2012).

Esta investigación respalda la utilidad de aplicar principios ergonómicos de amplio 
alcance, junto con un enfoque participativo, con el fin de mejorar los sistemas de trabajo en el sector de la salud. Evidencia de esto fueron los 22 puntos de mejoras identificados, los cuales constituyen una contribución a la seguridad del paciente y seguridad laboral desde una perspectiva integral.

Es importante destacar que el proceso de intervención ergonómica puede, en sí mismo, generar conciencia y educar a los profesionales de la salud y otros diversos actores sobre la importancia de adoptar la ergonomía para mejorar la seguridad del paciente. La concienciación y la educación son esenciales en América Latina, donde se ha reconocido la falta de aplicación sistemática de la ergonomía a los sistemas sanitarios (Aceves-González et al., 2020). Iniciativas similares de investigación-acción podrían servir como fuerza de movilización dentro de la región latinoamericana, para promover la simbiosis entre la ergonomía y la seguridad del paciente, siguiendo la larga tradición de uso de este enfoque (Helali, 2009). Es por ello por lo que visibilizar este tipo de iniciativa es un elemento clave para ganar aceptación y movilización internacional (Aceves-González et al., 2020; Torres et al., 2021).

\section{Límites del estudio y perspectiva futura}

Este estudio constituye un ejemplo de cómo analizar, desde la perspectiva de la ergonomía, áreas y procesos del sector sanitario. La intención principal del estudio presentado en este artículo es mostrar cómo el uso de listas de verificación ergonómica prácticas, bajo un enfoque participativo, puede ser de gran utilidad para los trabajadores y profesionales interesados en mejorar los sistemas de salud y la seguridad del paciente. Próximas investigaciones pueden extenderse a otras áreas del hospital, involucrar a otros actores del sistema de trabajo, como los directivos, administradores, los pacientes y familiares, entre otros. También se recomienda ampliar el alcance de este tipo de estudios hasta la fase de implementación de las mejoras, lo que requiere de mayor tiempo, recursos y compromiso entre los diferentes actores involucrados.

En el futuro, para aumentar el impacto de investigaciones similares, se debe adoptar una visión holística de los sistemas de salud. En este sentido, el modelo de integración de la ergonomía en los sistemas de salud o modelo del muro de protección (Model for the Integration of Ergonomics in Healthcare Systems), propuesto recientemente (Rodríguez \& Hignett, 2021), puede servir de marco de referencia para lograr que la aplicación de la ergonomía ocurra a diferentes niveles (micro, meso y macro) e impacte variables relevantes de los sistemas de salud (ej. capacidad, cobertura, integridad, robustez y resiliencia).

\section{Conclusiones}

En el contexto de los países en vías de desarrollo, la aplicación de los principios de la ergonomía mediante el uso de listas de verificación fáciles de usar, en combinación con un enfoque participativo, puede impulsar la integración de la ergonomía en el sector sanitario y de esta manera contribuir a mejorar la seguridad del paciente y la seguridad laboral. Además, promueve, entre los profesionales de la salud, la necesidad de incorporar los principios de 
ergonomía en los sistemas de trabajo donde se desempeñan.

El estudio presentado en este artículo constituye una novedad en el ecosistema sanitario colombiano y se espera abra las puertas a futuros desarrollos. También apoya el establecimiento de una estrategia nacional más amplia para la mejora de la seguridad del paciente y las condiciones laborales de los trabajadores de la salud, que incluya la ergonomía como un pilar fundamental. 


\section{Referencias}

Aceves-González, C., Rodríguez, Y., Escobar-Galindo, C. M., Pérez, E., Gutiérrez-Moreno, B., Hignett, S., \& Lang, A. R. (2020). Frontiers in human factors: Integrating human factors and ergonomics to improve safety and quality in Latin American healthcare systems. International Journal for Quality in Health Care, 33(Supl. 1), 45-50. https://doi.org/10.1093/intqhc/mzaa135

Aranaz-Andrés, J. M., Aibar-Remón, C., Limón-Ramírez, R., Amarilla, A., Restrepo, F. R., Urroz, O., ... Larizgoitia, I. (2011). Prevalence of adverse events in the hospitals of five Latin American countries: Results of the 'Iberoamerican study of adverse events' (IBEAS). BMJ Quality \& Safety, 20(12), 1043. https://doi.org/10.1136/bmjqs.2011.051284

Blouin, A. S., Smith-Miller, C. A., Harden, J., \& Li, Y. (2016). Caregiver fatigue: Implications for patient and staff safety, part 1. The Journal of Nursing Administration, 46(6), 329-335. https://doi.org/10.1097/nna.0000000000000353

Budnick, P., Kogi, K., \& O’Neill, D. (2012). Examples of practical ergonomics in industrially developing countries. Ergonomics in Design: The Quarterly of Human Factors Applications, 2O(4), 5-11. https://doi.org/10.1177/1064804612460041

Carayon, P., Xie, A., \& Kianfar, S. (2014). Human factors and ergonomics as a patient safety practice. BMJ Quality \& Safety, 23(3), 196-205. https://doi.org/10.1136/bmjqs-2013-001812

Donaldson, L. (2002). An organisation with a memory. Clinical Medicine Journal, 2(5), 452-457. https://doi.org/10.7861/clinmedicine.2-5-452

Helali, F. (2009). Using ergonomics checkpoints to support a participatory ergonomics intervention in an industrially developing country (IDC)-A case study. International Journal of Occupational Safety and Ergonomics, 15(3), 325-337. https://doi.org/10.1080/10803548.2009.11076811

Hignett, S., Carayon, P., Buckle, P., \& Catchpole, K. (2013). State of science: Human factors and ergonomics in healthcare. Ergonomics, 56(10), 1491-1503. https://doi.org/10.1080/00140139.2013.822932

Holden, R. J., Carayon, P., Gurses, A. P., Hoonakker, P., Hundt, A. S., Ozok, A. A., \& RiveraRodriguez, A. J. (2013). SEIPS 2.0: A human factors framework for studying and improving the work of healthcare professionals and patients. Ergonomics, 56(11), 1669-1686. https://doi.org/10.1080/00140139.2013.838643

Huck-Soo, L., \& Richardson, S. (2012). Ergonomics in industrially developing countries: A literature review. Journal of Human Ergology, 41(1-2), 1-16. https://doi.org/10.11183/jhe.41.1

International Ergonomics Association (IEA) (2000). Human factors/ergonomics (HF/E). https://iea.cc/what-is-ergonomics/

International Ergonomics Association (IEA) (2017). Ergonomic checkpoints in health care work. In: IEA Press. Human Ergology Society and International Ergonomics Association, pp. 1168.

International Labour Organization \& International Ergonomics Association (2010). Ergonomic checkpoints: Practical and easy-to-implement solutions for improving safety, health and working conditions. https://www.ilo.org/global/publications/ilo-bookstore/orderonline/books/WCMS 120133/lang--en/index.htm

Kogi, K., Sano, Y., Yoshikawa, T., \& Yoshikawa, S. (2019). The design and use of ergonomic checkpoints for health care work. Paper presented at the Proceedings of the 20th Congress of the International Ergonomics Association (IEA 2018), Cham. 
Machado-Alba, J. E., Moncada, J. C. y Moreno-Gutiérrez, P. A. (2016). Errores de medicación en pacientes atendidos en servicios ambulatorios de Colombia, 2005-2013. Biomédica, 36(2), 251-257. https://doi.org/10.7705/biomedica.v36i2.2693

Makary, M. A., \& Daniel, M. (2016). Medical error-the third leading cause of death in the US. $B M J, 353$, i2139. https://doi.org/10.1136/bmj.i2139

Moscoso, S., Parra, C., Ángel, N. y Padilla, M. (2015). Prevención de errores de medicación en la dispensación de medicamentos a pacientes ambulatorios, Colombia junio 2014-junio 2015. $\begin{array}{llll}\text { Vitae, } & 22(\text { Supl. } & 1 \text { ), S94-S95. }\end{array}$ https://revistas.udea.edu.co/index.php/vitae/article/view/2489o/20266

National Health Service (2018). Developing a patient safety strategy for the National Health Service. Proposals for consultation. In: NHS Improvement, pp. 1-23. London.

Organisation for Economic Co-operation and Development (OECD) (2018). Health expenditure and financing. In OECD.Stat. https://stats.oecd.org/

Pisters, P. (2015). University Health Network pioneers discussion about medical errors in Canada.

https://www.uhn.ca/corporate/News/Pages/Dr Peter Pisters looks ahead with new vis ion for UHN.aspx

Rodríguez, Y., \& Hignett, S. (2021). Integration of human factors/ergonomics in healthcare systems: A giant leap in safety as a key strategy during Covid-19. Human Factors and Ergonomics in Manufacturing \& Service Industries, $1-7$. https://doi.org/10.1002/hfm.20907

Torres, Y., Rodríguez, Y., \& Buitrago, N. R. (2021, 2021//). Application of the ergonomic checkpoints in health care work: A case from an inpatient service unit of an educational hospital in Colombia. Paper presented at the Proceedings of the 21st Congress of the International Ergonomics Association (IEA 2021), Cham.

World Health Organization (2017). Patient safety: Making health care safer. https://apps.who.int/iris/bitstream/handle/10665/255507/WHO-HIS-SDS-2017.11eng.pdf; sequence $=1$

World Health Organization (2019a). Patient safety: A global health priority. https://www.who.int/patientsafety/policies/global-health-priority/en/

World Health Organization (2019b). Patient safety: Data and statistics. http://www.euro.who.int/en/health-topics/Health-systems/patient-safety/data-andstatistics 